\title{
ESTUDIO OBSERVACIONAL DEL SEXISMO EN LA EDUCACIÓN FÍSICA DEL SUR DE ESPAÑA
}

\author{
OBSERVATIONAL STUDY ON SEXISM IN PHYSICAL EDUCATION IN \\ SOUTHERN SPAIN
}

ESTUDO OBSERVACIONAL DO SEXISMO NA EDUCAÇÃO FÍSICA DO

SUL DA ESPANHA

\author{
Óscar DelCastillo-Andrés*, María del Carmen Campos-Mesa*, \\ Carolina Castañeda*, Gloria González ${ }^{*}$
}

\begin{abstract}
Palabras clave Educación y Entrenamiento Físico. Investigación Cualitativa. Identidad de Género. Educación Primaria y Secundaria.

Resumen: Esta investigación analiza el comportamiento de 240 estudiantes para determinar cómo se establecen conductas sexistas entre el alumnado de Educación Secundaria, en Sevilla (España), dentro de la sesión práctica de Educación Física. Se categorizaron códigos descriptivos de observación. Se identificaron tres categorías relacionadas con conductas sexistas: implicación en la tarea, solicitación de información adicional y aceptación de tareas. Se utilizó una escala de observación para registrar las frecuencias. El test Mann-Whitney estableció una $p<0.05$ para rechazar la hipótesis nula. Aparecen comportamientos sexistas en la implicación en la tarea. No aparece discriminación en la solicitación de información adicional y aceptación de tareas.
\end{abstract}

Keywords Physical Education and Training. Qualitative Research. Gender Identity. Primary and Secondary Education.

\section{Palavras-chave Educação Física e treinamento. Pesquisa qualitativa. Identidade de gênero. Ensino fundamental e médio.}

Abstract: This study analyzed the behavior of 240 students to determine how sexist behavior is established among Secondary Education students in Seville (Spain), during the practical session of Physical Education. Descriptive codes were set for observation. Three categories related to sexist behavior were identified: involvement in the task; requesting additional information; and task acceptance. An observation scale was used to record frequencies. The Mann-Whitney test established $p<0.05$ to reject the null hypothesis. Sexist behaviors emerge in involvement in task. No discrimination appears in requesting additional information and task acceptance.

Resumo: Esta pesquisa analisa o comportamento de 240 estudantes para determinar como se estabelecem comportamentos sexistas entre os alunos de Educação Física da Educação Secundária em Sevilha (Espanha). Categorizaram-se códigos descritivos de observação. Foram identificadas três categorias relacionadas ao comportamento sexista: envolvimento na tarefa, solicitação de informação adicional e aceitação de tarefas. Uma escala de observação foi utilizada para registrar as frequências. $O$ teste de Mann-Whitney establece uma $p<0,05$ para rejeitar a hipótese nula. Encontrouse comportamento sexista no envolvimento na tarefa. Não aparece discriminação na solicitação de informação adicional e aceitação de tarefas.
* Universidad de Sevilla. Sevilla. España.

E-mail: ocastillo@us.es

Recebido em: 18-05-2015 Aprovado em: 23-08-2015 (c) (1) (8) Licence 


\section{INTRODUCCIÓN ${ }^{1}$}

Los procesos de socialización de género ${ }^{2}$ de nuestro alumnado a través de la Educación Física (EF), área de conocimiento influyente en la transmisión de valores, son un tema de gran actualidad e interés, como así lo demuestra la amplia producción científica que existe al respecto, tanto en el contexto español (ej.: DELCASTILLO-ANDRÉS et al., 2014, MORA; CRUZ; SOUSA, 2013, VALDIVIA-MORAL et al, 2012), como en el entorno internacional (ej.: CHALABAEV et al., 2013, PRIES DEVIDE et al., 2011, PREWITTFREILINO; CASWELL; LAAKSO, 2012). En esta misma línea, y a modo de ejemplo, la revista Movimento presenta más de 200 artículos relacionados con los citados procesos de socialización de género.

El tema de la inequidad de género tiene un gran interés educativo en España, plasmado en los objetivos de la Ley Orgánica para la Mejora de la Calidad Educativa (LOMCE, 2013). En el sur de España, en Andalucía, el referente es el I Plan Estratégico para la Igualdad de Mujeres y Hombres en Andalucía (INSTITUTO ANDALUZ DE LA MUJER, 2010). La sociedad española actual ha establecido la escolarización mixta en las escuelas públicas y privadas para los estudiantes hasta los 16 años de edad. Este modelo de escolarización mixta ha sido diseñado para fomentar un sistema no sexista entre alumnas y alumnos (LOMCE, 2013). Bajo esta premisa, varios autores han adoptado enfoques coeducativos con el objetivo de eliminar las diferencias de género en el aula (ALBERT LÓPEZ; CAMERINO FOGUET; CASTAÑER BALCELLS, 2015, GIL-MADRONA et al., 2014). Sin embargo, a pesar de estas directrices, el profesorado de EF en España presenta una utilización generalizada de los paradigmas tradicionales de enseñanza, lo cual conlleva estructuras metodológicas que fomentan la discriminación de género ${ }^{3}$ del alumnado (BROWN; CHU, 2012, DELCASTILLO-ANDRÉS; CAMPOS-MESA; CASTAÑEDA, 2015). Estudios recientes han demostrado en España que el profesorado, a través de elementos vinculados al estilo de enseñanza, contribuye a las desigualdades de género discriminando al femenino (DELCASTILLO-ANDRÉS; CORRAL PERNÍA, 2011). Fuera de España, en los Estados Unidos, Leaper y Brown (2008) o Davis (2003) sugieren que los profesores y profesoras se comportan de manera diferente en el aula, y esta diferencia se refleja en una mayor participación de los alumnos. En la misma línea, Duffy, Warren y Walsh (2001) en Canadá y Lentillon, Cogérino y Kaestner (2006) en Francia, han demostrado que el profesorado proporciona a las alumnas, menos información, peor material o espacios de juego menos amplios, entre otros. Asimismo, DelCastillo-Andrés et al. (2012, 2014) o Valdivia-Moral et al. (2012), han analizado la discriminación de las alumnas a través de dimensiones como el lenguaje o la información que utiliza el profesorado, los libros de texto, el Currículum, etc.

Pero existen elementos dentro de la lección práctica de EF que requieren una observación más detallada para que puedan proporcionar una imagen más completa del comportamiento sexista del alumnado en el aula (SANTOS, 2010). Estamos convencidos de que se necesita más investigación cualitativa para determinar la relación entre la implicación del alumnado en la tarea, la solicitación de información adicional y la aceptación de tareas y su relación con

1 No existe un potencial conflicto de intereses para este estudio

2 Se entiende por género el conjunto de expectativas y comportamientos que una sociedad asigna y espera de la persona en función de su sexo masculino o femenino. 
la equidad de género. Suponemos que estas dimensiones tienen un efecto importante en la configuración de los estereotipos de género durante el desarrollo de las actividades de clase de EF.

Para entender la relación de las anteriores variables con el establecimiento de los estereotipos y roles de género en este contexto de investigación, el posicionamiento epistemológico de la Teoría Ecológica de Bronfenbrenner (1999), permite extraer información etnográfica única sobre el comportamiento del alumnado de EF en términos de su género. En base a dicha teoría, se puede establecer cómo se desarrollan las relaciones de género en un contexto educativo concreto partiendo del análisis de su microsistema (en este caso particular sobre los patrones de actividades y relaciones interpersonales que el alumnado experimenta en el aula) sobre el cual se establecerán diferentes roles de género; este nivel "micro" se expresa en el discurso y en las formas comportamentales establecidas por género. Posteriormente, estos roles de género serán reforzados a nivel mesosistémico (escuela, hogar, etc.), hasta llegar a la estructura macrosistémica en la cual encontraremos consolidados los valores, creencias e ideologías que determinan cómo entiende una cultura concreta las relaciones de género.

Al respecto, este trabajo muestra los resultados de un estudio cualitativo observacional sobre el establecimiento de conductas sexistas entre el alumnado del nivel de Educación Secundaria Obligatoria (ESO), en centros educativos públicos de Sevilla (España), dentro de la lección práctica de EF. Se trata de evaluar si existe discriminación de género entre alumnas y alumnos, en cuanto a las dimensiones implicación en la tarea, solicitación de información adicional y aceptación de tareas, en las actividades propuestas por el profesorado. La intención fue establecer una relación significativa de sexismo ${ }^{4}$ entre las dimensiones anteriores, el género del profesorado, el género del alumnado, el estilo de enseñanza, la unidad didáctica y el curso. Este estudio puede proporcionar al profesorado una herramienta de diagnóstico fiable sobre las interacciones sexistas del alumnado, lo cual dará la posibilidad de evitar las desigualdades de género dentro de las lecciones prácticas de EF.

\section{LA IMPLICACIÓN DEL ALUMNADO EN LA TAREA}

Con la implicación del alumnado en la tarea se pretende establecer la disposición del alumnado hacia la realización de una tarea. Realizada una profunda revisión bibliográfica sobre esta dimensión, no se han encontrado estudios que se ajusten al perfil que aquí se presenta, por lo que supone un aporte novedoso a la literatura científica sobre estudios de género. Para estructurar el marco teórico de esta dimensión se plantea el análisis de aquellos elementos que se creen relevantes para justificar si un alumno o alumna se está implicando en la tarea propuesta.

En primer lugar, es importante conocer el perfil dispositivo-motivacional de nuestro alumnado para establecer qué actitudes puede presentar en una sesión práctica de EF. Para ello partimos de los estudios de Griffin $(1984,1985)$ quién determinó las formas de implicación del alumnado. Como resultado estableció seis tipos de comportamientos para las alumnas ${ }^{5}$ y cinco

\footnotetext{
4 Discriminación de personas de un sexo por considerarlo inferior al otro.

5 Athletes (presenta alta participación y rendimiento en las tareas), junior varsity players (participación temporal y menor rendimiento que las anteriores), cheerleaders (bajo rendimiento y alta participación y entusiasmo), lost souls (muy bajo rendimiento y participación muy ocasional invisible), femme fatales (participación activa en presencia de los chicos) y System Beaters (evita participar con justificación médica, paterna, etc.).
} 
tipos para los alumnos 6 . Realizando una síntesis y adaptación de los mismos, para ajustarlos a nuestro contexto de actuación, y apoyándonos, del mismo modo, en los propuestos por Carlson (1995) y Bennett (2000), definimos para nuestra investigación tres tipos de comportamientos relacionados con la cooperación del alumnado en actitudes equitativas respecto al género: coopera con los dos géneros, no coopera con los dos géneros e invisible ${ }^{7}$ (ver Apéndice). Cabe señalar que algunos comportamientos establecidos por los anteriores autores no han sido tenidos en cuenta en esta investigación debido a que los perfiles estaban relacionados fundamentalmente con el rendimiento físico del alumnado y no con sus actitudes.

En la línea del análisis de las variables que favorecen la implicación del alumnado en las clases de EF se encuentran los actuales trabajos en España de Abós et al. (2015), Cañabate Ortíz et al. (2014), Granero-Gallegos y Baena-Extremera (2014) o DelCastillo-Andrés, Campos y Ries (2013), donde se señalan los diferentes motivos y niveles de implicación del alumnado en las clases de EF en relación a parámetros como, por ejemplo, un mayor climatarea de las sesiones prácticas para implicar a las alumnas. A esta conclusión se suma, en el ámbito internacional, la de Carlson (1995), quien señala como factor determinante de la poca implicación de las alumnas en la tarea, la frecuente orientación de las clases hacia la competición. Otros dos elementos actitudinales que se proponen en esta investigación son el uso de ropa adecuada a la actividad y la aportación de ideas vinculadas a un mejor desarrollo de las tareas propuestas. Esta relación actitudinal puede asociarse a otros predictores de comportamiento, como por ejemplo, el tipo de contenido a desarrollar (físico o técnico), para establecer qué posibilidades de colaboración-cooperación se espera encontrar entre el alumnado (GRANERO-GALLEGOS; BAENA-EXTREMERA, 2014, MARTOS GARCIA et al., 2014).

\section{LA SOLICITACIÓN DE INFORMACIÓN ADICIONAL}

Tras una exhaustiva revisión bibliográfica de los fundamentos teóricos relacionados con esta dimensión, no se encuentran estudios relacionados con la misma. Por tanto, se ve la necesidad de definir esta dimensión.

La dimensión Solicitación de información adicional, pretende establecer si son los alumnos o las alumnas quienes presentan mayor interés por una ampliación de la información sobre la ya proporcionada por el profesorado. Teniendo esto en cuenta, se define esta dimensión como aquella información suplementaria, relacionada con la tarea que se está realizando y que el alumnado, de forma espontánea, requiere del profesorado. Esta información puede ser una demanda inicial o una ampliación de la explicación ya llevada a cabo y puede ser realizada en cualquier momento de la sesión práctica.

De constatarse un mayor interés por parte de uno de los géneros, los datos obtenidos permitirán establecer una línea de trabajo que permita superar la discriminación de género

\footnotetext{
6 Machos (presenta alta participación y rendimiento en las tareas; liderazgo), junior machos (menor tamaño físico y rendimiento en la tarea que los anteriores), nice guys (participación media y cooperación con las chicas), invisible players (participación casi nula y muy ocasional y muy bajo rendimiento) y wimps (baja participación y preferencia por deportes femenimos).

7 En la línea de los estudios de Griffin $(1984,1985)$, definimos al alumnado invisible, como aquel que no habla con compañeros o profesorado, no manipula el material necesario, habitualmente se encuentra en posiciones periféricas a la tarea y, por último, el resto de los compañeros lo ignoran cuando lo ven.
} 
planteada. Así mismo, se pretende relacionar, en posteriores trabajos, la solicitación de información adicional con otras dimensiones planteadas ya por DelCastillo-Andrés et al. (2012, 2014), como por ejemplo, el uso del lenguaje o el canal de comunicación empleado por el profesorado, lo cual permitirá obtener información significativa sobre dichas relaciones.

\section{LA ACEPTACIÓN DE TAREAS}

La dimensión Aceptación de tareas pretende establecer la respuesta de aceptación 0 rechazo que manifiesta uno y otro género ante las diferentes actividades (de contenido físico o técnico) que son propuestas en una sesión práctica. Al igual que en el epígrafe anterior, la falta de estudios o referencias en relación a esta dimensión nos lleva a definir el término. Se establece la aceptación de tareas como la aprobación por parte del alumno o la alumna hacia la tarea propuesta por el profesor o la profesora. Esto implica que, posteriormente a la información impartida por el profesorado, no exista ninguna discusión evasiva o gesto de desagrado sobre la tarea que se debe realizar.

La información obtenida de esta dimensión será significativa en cuanto a la posibilidad de relacionarla con dimensiones como la implicación en la tarea y la solicitación de información adicional.

Consecuentemente, de acuerdo con la literatura revisada y con los nuevos planteamientos de las dimensiones aquí definidas se presume que, en los Institutos de Enseñanza Secundaria españoles estudiados, existe transmisión del sexismo en favor de los alumnos, en cuanto a la implicación del alumnado, la solicitación de información y la aceptación de tareas. De constatarse este hecho, supondría la necesidad de llevar a cabo una reorientación de las sesiones prácticas de EF hacia nuevas metodologías de trabajo que permitan lograr cambios en la conducta del alumnado hacia nuevos hábitos, valores y creencias orientadas a la equidad de género (DYSON, LINEHAN; HASTIE, 2010, ACKER et al., 2010).

En definitiva, el propósito de este estudio es examinar las acciones de los alumnos y las alumnas para evaluar cómo el sexismo es transmitido a través de la participación del alumnado. Para ello se plantean tres hipótesis principales:

1. Los alumnos se implican más que las alumnas en la realización de Habilidades deportivas propuestas por un profesor que utiliza el Mando directo para impartir un contenido técnico en primer curso (Hipótesis 1).

2. Los alumnos solicitan más información adicional que las alumnas a los profesores cuando trabajan Habilidades deportivas de contenido técnico a través de Mando directo en primer curso (Hipótesis 2).

3. Las alumnas rechazan las tareas propuestas por los profesores cuando el contenido a desarrollar en la sesión práctica de EF es Habilidad deportiva de contenido físico impartida a través de Mando directo en primer curso (Hipótesis 3). 


\section{MÉTODO}

El método ha sido desarrollado en anteriores publicaciones (DELCASTILLO-ANDRÉS et al., 2012, 2014).

En la presente investigación los datos se han obtenido de dos centros públicos de Enseñanza Secundaria Obligatoria de Sevilla (sur de España) con un nivel socioeconómico medio-bajo. La aplicación de una Escala de observación permitió analizar la participación del alumnado en el aula y recoger datos sobre conductas sexistas en relación al género. Se registraron frecuencias de comportamiento en relación a la implicación del alumnado en la tarea, la solicitación de información adicional y la aceptación de tareas. Todos los análisis se realizaron con el paquete estadístico para Ciencias Sociales (SPSS, versión 19.0 para Windows, SPSS Inc., Chicago, IL). Se fijó el nivel de significación en $p<0.05$. La normalidad de las variables se evaluó mediante la prueba de Kolmogorov-Smirnov, la cual demostró la falta de normalidad en la distribución de las dimensiones.

\section{PARTICIPANTES}

En la investigación participaron 240 estudiantes (129 hombres y 111 mujeres), con edades comprendidas entre 12 y $14(M=13.1, S D=0.75)$. La ratio de estudiantes por profesorado fue de 30:1. Se informó a los padres y a las madres de los estudiantes de que iban a ser grabados en audio y video. Se obtuvo el consentimiento de todos los participantes. A los estudiantes se les aseguró la confidencialidad de las grabaciones.

La muestra de profesores estuvo formada por dos hombres y dos mujeres seleccionadas de forma incidental (SALKIND, 1999). Todos ellos eran españoles, blancos y de clase social media. Su edad media fue de 37.5 años $(S D=1.25)$. El profesorado fue informado de la metodología que se iba a utilizar para recoger datos (grabación de audio y vídeo, así como el registro en la escala de observación por los observadores in situ en cada una de las sesiones). El profesorado no había recibido instrucciones específicas sobre la equidad de género y todos dieron su consentimiento por escrito para ser observados.

\section{PROCEDIMIENTO}

Para llevar a cabo el estudio observacional se desarrolló un sistema de categorías (CASTELLANO PAULIS; HERNÁNDEZ MENDO, 2015). El esquema de codificación fue revisado y modificado en varias ocasiones hasta establecer una agrupación de códigos similares que detectaran coincidencias y eliminaran redundancias. Los códigos se clasificaron y categorizaron hasta establecer tres temas relacionados con la formación de sexismo en el alumnado en las sesiones prácticas de EF. Como resultado surgieron tres categorías como elementos relevantes en la discriminación de género del alumnado: la implicación del alumnado en la tarea, la solicitación de información adicional y la aceptación de tareas.

Los datos fueron recogidos por nueve observadores. Entre ellos se encontraba el investigador (masculino) y otros ocho observadores (cuatro masculinos y cuatro femeninos). Se registró la frecuencia de los comportamientos en la escala de observación durante 48 sesionesprácticas de EF (dos sesiones de 1 hora a la semana). Se registraron un total de 12 
horas de clase para cada profesor (6 horas de Habilidades deportivas y 6 horas de Expresión corporal).

\section{INSTRUMENTO}

La hoja de codificación es parte de un instrumento llamado Escala de Observación de la Equidad Educativa del alumnado en materia de género (DELCASTILLO-ANDRÉS, 2009).

La fiabilidad de este estudio observacional se determinó con el Índice Kappa, el cual fue establecido para nueve observadores trabajando de forma independiente. Se establecieron valores inter observadores para el Índice Kappa de 0.89 en la implicación del alumnado en la tarea, 0.75 en la solicitación de información adicional y 0.91 en la aceptación de tareas. La fiabilidad no demostró sesgo debido al género de los observadores. Por lo tanto, la fiabilidad del procedimiento de codificación es adecuada y se utilizaron estas categorizaciones para los análisis.

\section{RESULTADOS}

Para comparar las dimensiones independientes (género del profesorado, género del alumnado, unidad didáctica, curso y estilo de enseñanza), con las dimensiones dependientes (implicación del alumnado en la tarea, solicitación de información adicional y aceptación de tareas) se utilizó la prueba de Kruskal-Wallis. Así mismo se aplicaron, a los datos que mostraron significatividad, los estadísticos descriptivos media y desviación estándar.

Nuestras predicciones fueron probadas mediante la observación de 240 estudiantes y cuatro profesores de EF con las características de clase que se muestran en la Tabla 1.

Tabla 1 - Ratio de estudiantes por curso y género del profesorado

\begin{tabular}{rcccc}
\hline & $\begin{array}{c}\text { Profesor 1 } \\
\text { (Hombre) }\end{array}$ & $\begin{array}{c}\text { Profesor 2 } \\
\text { (Hombre) }\end{array}$ & $\begin{array}{c}\text { Profesor 3 } \\
\text { (Mujer) }\end{array}$ & $\begin{array}{c}\text { Profesor 4 } \\
\text { (Mujer) }\end{array}$ \\
\hline Curso 1(Chicos $n=65$; Chicas $n=55)$ & & & & \\
Chicos, $n$ & 14 & 10 & 23 & 18 \\
Chicas, $n$ & 16 & 20 & 7 & 30 \\
Total, $n$ & 30 & 30 & 30 & 12 \\
\hline Curso 2 (Chicos $n=64$; Chicas $n=56)$ & & & & 12 \\
Chicos, $n$ & 19 & 13 & 20 & 30 \\
Chicas, $n$ & 11 & 17 & 10 & 30 \\
Total, $n$ & 30 & 30 & & 30
\end{tabular}

\section{IMPLICACIÓN DEL ALUMNADO EN LA TAREA}

Se encontraron diferencias significativas (tabla 2), en relación al género del profesorado, en la implicación en la tarea en cuanto a la cooperación y no cooperación con los dos géneros, la aportación de ideas y el uso de ropa deportiva. En relación a la unidad didáctica, es significativa la implicación en cuanto a la cooperación y no cooperación con los dos géneros, el alumnado invisible, la aportación de ideas y el uso de ropa deportiva. Según el estilo de enseñanza se pueden observar significatividad en la implicación en relación a la cooperación con los dos géneros. 
Tabla 2 - Análisis estadístico implicación en la tarea / Género del profesorado; Unidad didáctica; Estilo de enseñanza; Curso

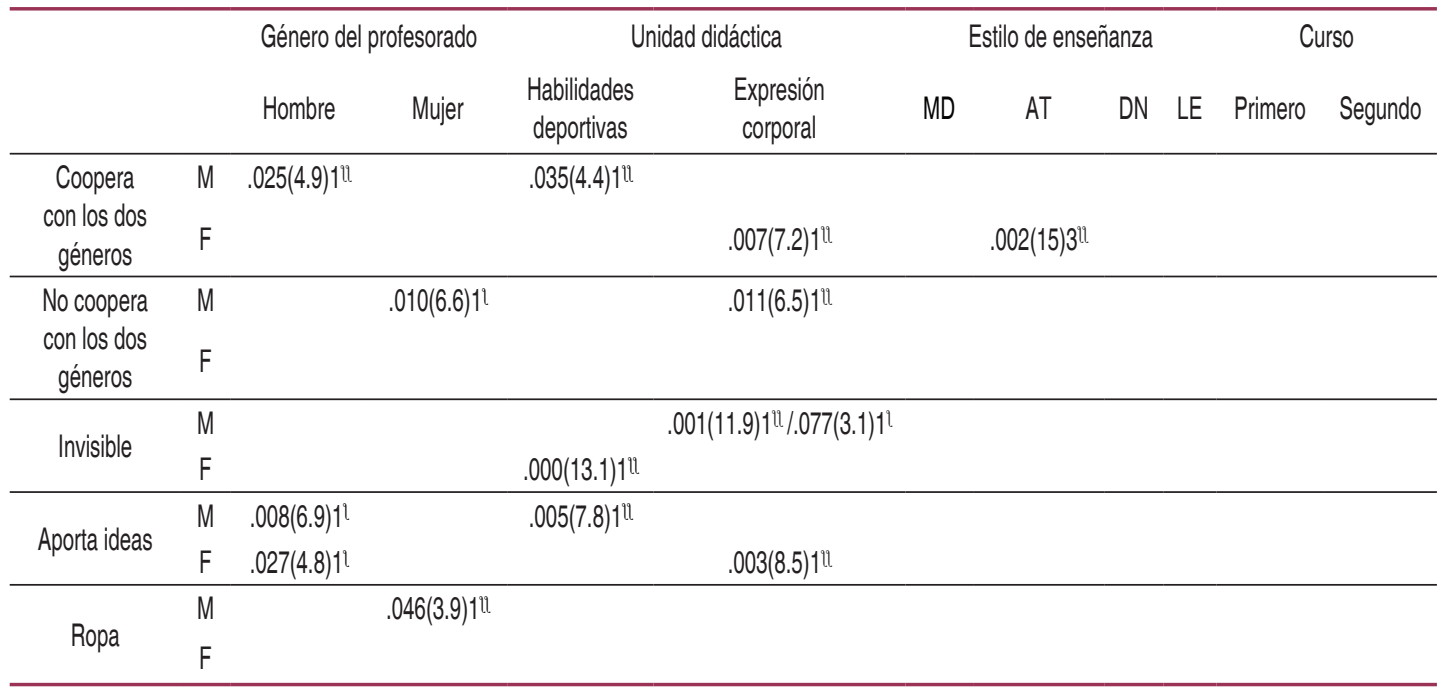

Nota: los datos son presentados en el formato $p\left(x^{2}\right) d f$ ( $p=$ significatividad; $d f=$ grados de libertad; $x^{2}=$ chi-cuadrado); "l contenido técnico; ' contenido físico; $\mathrm{M}$ = Alumnos; $\mathrm{F}$ = Alumnas; $\mathrm{MD}$ = Mando directo; $\mathrm{AT}$ = Asignación de tareas; $\mathrm{DN}$ = Diversificación de niveles; $\mathrm{LE}$ = Libre exploración.

Por su parte, los estadísticos descriptivos (tabla 3) mostraron que, los alumnos (coopera con los dos géneros y aporta ideas) y las alumnas (aporta ideas) se implican más cuando la sesión práctica la imparte un profesor. Por otro lado, los alumnos no se implican (no coopera con los dos génerosy no ropa deportiva) cuando la sesión es impartida por una profesora. Así mismo, los alumnos se implican (cooperan con los dos géneros y aportan ideas) si la unidad didáctica está relacionada con las Habilidades deportivas de contenido técnico, mientras que no se implican (no cooperan con los dos géneros o invisible) si está relacionada con la Expresión corporal. En cuanto a las alumnas, estas se implican (coopera con los dos géneros y aporta ideas) si la unidad didáctica es de Expresión corporal y contenido técnico y no se implican (invisible), cuando se trata de Habilidades deportivas de contenido técnico.Por último, las alumnas presentan mayor implicación (cooperación con los dos géneros) cuando el estilo de enseñanza es la asignación de tareas para actividades de contenido técnico.

Tabla 3 - Estadísticos descriptivos implicación en la tarea / Género del profesorado; Unidad didáctica; Estilo de enseñanza; Curso

\begin{tabular}{|c|c|c|c|c|c|c|c|c|c|c|}
\hline \multirow[b]{2}{*}{ Hombre } & & \multicolumn{2}{|c|}{ Género del profesorado } & \multicolumn{2}{|c|}{ Unidad didáctica } & \multicolumn{4}{|c|}{ Estilo de enseñanza } & \multirow{2}{*}{$\begin{array}{l}\text { Curso } \\
\text { Segundo }\end{array}$} \\
\hline & & Mujer & $\begin{array}{l}\text { Habilidades } \\
\text { deportivas }\end{array}$ & $\begin{array}{l}\text { Expresión } \\
\text { corporal }\end{array}$ & MD & AT & DN & LE & Primero & \\
\hline \multirow{2}{*}{$\begin{array}{l}\text { Coopera con los } \\
\text { dos géneros }\end{array}$} & M & $1.67^{\star}(1.85)^{\mathrm{ul}}$ & $0.63(1.05)^{\mathrm{tl}}$ & $1.71^{\star}(1.92)^{\mathrm{th}}$ & $0.58(0.88)^{t u}$ & & & & & \\
\hline & $\mathrm{F}$ & & & $0.96(1.57)^{\mathfrak{l l}}$ & $2.58^{*}(2.24)^{11}$ & $0.23(1.08)^{1 \mathrm{t}}$ & $1^{*}(-)^{2}$ & $0(-)^{2}$ & $0(0)^{2}$ & \\
\hline \multirow{2}{*}{$\begin{array}{l}\text { No coopera con } \\
\text { los dos géneros }\end{array}$} & M & $0(0)^{2}$ & $0.71^{*}(1.51)^{\mathrm{q}}$ & $1.13(1.94)^{\mathfrak{t h}}$ & $2.88^{*}(2.49)^{\natural 1}$ & & & & & \\
\hline & $\mathrm{F}$ & & & & & & & & & \\
\hline \multirow[t]{2}{*}{ Invisible } & M & & & 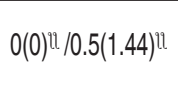 & $\begin{array}{l}0.46^{\star *}(1.66)^{\natural} \\
12.13^{\star}(2.54)^{\natural \mathrm{l}}\end{array}$ & & & & & \\
\hline & $\mathrm{F}$ & & & $2.54^{\star \star}(2.46)^{11}$ & $0.25(0.73)^{\mathfrak{l t}}$ & & & & & \\
\hline \multirow{2}{*}{ Aporta ideas } & M & $0.96^{*}(1.12)^{\mu l}$ & $0.21(0.58)^{\mathfrak{}}$ & $0.92^{\star}(0.97)^{11}$ & $0.25(0.84)^{11}$ & & & & & \\
\hline & $\mathrm{F}$ & $0.88^{\star}(1.36)^{\mu \mathrm{u}}$ & $0.17(0.56)^{t t}$ & $0.04(0.20)^{1 \mathrm{u}}$ & $1^{\star}(1.38)^{\mathfrak{n}}$ & & & & & \\
\hline \multirow{2}{*}{ Ropa } & M & $0.63(0.64)^{n}$ & $1.13^{*}(0.90)^{11}$ & & & & & & & \\
\hline & $\mathrm{F}$ & $1.58(0.77)^{\mathfrak{t l}}$ & $1.29(0.99)^{\mathrm{u}}$ & & & & & & & \\
\hline
\end{tabular}




\section{SOLICITACIÓN DE INFORMACIÓN ADICIONAL}

En relación al género del profesorado se encontraron diferencias significativas en la solicitación de información adicional por parte de los alumnos $\chi 2(d f=1, N=48)=5.625, p=$ .018 y las alumnas $\chi 2(d f=1, N=48)=9.020, p=.003$. Para la Unidad didáctica es significativa la solicitación de información y el género del alumnado $\chi 2(\mathrm{df}=1, \mathrm{~N}=48)=4.459, p=.035$.

Los estadísticos descriptivos (tabla 4) mostraron que, tanto las alumnas, como los alumnos, solicitan información cuando la sesión práctica la imparte un profesor y es durante el desarrollo de Habilidades deportivas cuando los alumnos solicitan más información adicional al profesorado.

Tabla 4 - Estadísticos descriptivos solicitación de información adicional / género del profesorado; unidad didáctica

\begin{tabular}{|c|c|c|c|c|}
\hline & \multicolumn{2}{|c|}{ Sexo del profesorado } & \multicolumn{2}{|c|}{ Unidad didáctica } \\
\hline & Hombre & Mujer & Habilidades deportivas & Expresión corporal \\
\hline Alumno & $4.50^{\star}(2.97)^{\text {ใน }}$ & $2.75(3.45)^{\mathfrak{4}}$ & $4.58^{\star}(3.25)^{\mathfrak{\imath}}$ & $2.67(3.14)^{\mathfrak{t}}$ \\
\hline Alumna & $4.96^{*}(5.47)^{\text {tน }}$ & $1.46(1.76)^{\natural \imath}$ & & \\
\hline
\end{tabular}

Nota: los datos son presentados en el formato $M(S D)$ ( $M=$ media aritmética; $S D=$ desviación típica); 'll contenido técnico; ' c contenido físico; ${ }^{*} p<.05$.

\section{ACEPTACIÓN DE TAREAS}

Se encontraron diferencias significativas (tabla 5), en relación a la unidad didáctica, para los alumnos en la aceptación de tareas de contenido físico y técnico y rechazo para tareas de contenido técnico; en cuanto a las alumnas, estas han destacado significatividad en la aceptación de tareas físicas y en el rechazo de tareas de carácter técnico. Para el estilo de enseñanza, existe significatividad para las alumnas en cuanto al rechazo de tareas técnicas. El curso es significativo para las alumnas en cuanto a la aceptación de tareas de contenido físico.

Tabla 5 - Análisis estadístico aceptación de tareas / Unidad didáctica; Estilo de enseñanza; Curso

\begin{tabular}{|c|c|c|c|c|c|c|c|c|c|}
\hline & & \multicolumn{2}{|c|}{ Unidad didáctica } & \multicolumn{4}{|c|}{ Estilo de enseñanza } & \multicolumn{2}{|c|}{ Curso } \\
\hline & & Habilidades deportivas & $\begin{array}{c}\text { Expresión } \\
\text { corporal }\end{array}$ & MD & AT & DN & LE & Primero & Segundo \\
\hline \multirow{2}{*}{ Acepta } & M & $.000(19.04) 11 / .035(4.44) 1^{11}$ & & & & & & & \\
\hline & $\mathrm{F}$ & $.000(20.37) 11$ & & & & & & & $.043(4.07) 1^{2}$ \\
\hline \multirow{2}{*}{ Rechaza } & M & & $.009(6.73) 1^{\text {น }}$ & & & & & & \\
\hline & $\mathrm{F}$ & $.008(6.96) 1^{1 \mathrm{tl}}$ & & & $.032(8.77) 3^{11}$ & $.032(8.77) 3^{11}$ & & & \\
\hline
\end{tabular}

Nota: los datos son presentados en el formato $p\left(\mathrm{x}^{2}\right) d f$ ( $p=$ significatividad; $d f=$ grados de libertad; $\mathrm{x}^{2}=$ chi-cuadrado); "l contenido técnico; ' contenido físico; M = Alumnos; F = Alumnas; MD = Mando directo; AT = Asignación de tareas; DN = Diversificación de niveles; LE = Libre exploración.

Los estadísticos descriptivos (tabla 6) indican, para la variable unidad didáctica, una mayor aceptación de las tareas por parte de los alumnos, cuando estas están relacionadas con las Habilidades deportivas, tanto si su contenido es físico, como técnico. Por el contrario, si es de Expresión corporal, y está centrada sobre aspectos técnicos, las conductas de los alumnos hacia la tarea son de rechazo. Las alumnas aceptan en mayor medida las tareas relacionadas 
con las Habilidades deportivas de carácter físico, mientras que rechazan las mismas actividades si son de contenido técnico. Según el estilo de enseñanza, tanto en la aplicación de la Asignación de tareas, como en la Diversificación de niveles, posibilitan un mayor rechazo por parte de las alumnas hacia las tareas de contenido técnico. En relación al curso, son las alumnas de segundo curso quienes aceptan en mayor medida las tareas de contenido físico.

Tabla 6 - Estadísticos descriptivos aceptación de tareas / Unidad didáctica; Estilo de enseñanza; Curso

\begin{tabular}{|c|c|c|c|c|c|c|c|c|c|}
\hline & & \multicolumn{2}{|c|}{ Unidad didáctica } & \multicolumn{4}{|c|}{ Estilo de enseñanza } & \multicolumn{2}{|c|}{ Curso } \\
\hline & & Habilidades deportivas & Expresión corporal & MD & AT & DN & LE & Primero & Segundo \\
\hline \multirow{2}{*}{ Acepta } & M & $2.63^{\star *}(1.43)^{1} / 4.50^{\star}(1.25)^{1 \mathrm{tu}}$ & $0.88(1.03)^{\mathrm{t} / 3.63(1.63)^{1 \mathrm{t}}}$ & & & & & & \\
\hline & $\mathrm{F}$ & $2.71^{* *}(1.42)^{1}$ & $0.88(1.03)^{\mathrm{l}}$ & & & & & $1.33(1.23)^{1}$ & $2.25^{\star}(1.70)^{1}$ \\
\hline \multirow{2}{*}{ Rechaza } & M & $0.04(0.20)^{11}$ & $0.46^{*}(0.72)^{\natural น}$ & & & & & & \\
\hline & $\mathrm{F}$ & $0.42^{*}(0.50)^{1 \mathrm{u}}$ & $0.08(0.28)^{14}$ & $0.29(0.46)^{1 \mathrm{t}}$ & $1^{*}(-)^{u}$ & $1^{*}(-)^{\natural}$ & $0.07(0.25)^{11}$ & & \\
\hline
\end{tabular}

Nota: los datos son presentados en el formato $M(S D)$ ( $M=$ media aritmética; $S D=$ desviación típica); "l contenido técnico; ' contenido físico; $M$ = Alumnos; $\mathrm{F}=$ Alumnas; $\mathrm{MD}=$ Mando directo; $\mathrm{AT}=$ Asignación de tareas; $\mathrm{DN}=$ Diversificación de niveles; $\mathrm{LE}=$ Libre exploración; ${ }^{*} p<.05$; ${ }^{* *} p<.001$.

\section{DISCUSIÓN}

Este estudio describe las interacciones sexistas que se producen entre el profesorado y el alumnado durante el transcurso de una sesión práctica de EF en centros de enseñanza secundaria del sur de España. Los principales hallazgos son la evidencia de discriminación en la dimensión implicación del alumnado en la tarea y la no discriminación por género en la solicitación de información adicional y aceptación de tareas. La discusión de estas dimensiones será de carácter general, pero con referencias específicas a nuestros resultados.

Del mismo modo, este estudio ha demostrado que los alumnos y las alumnas actúan de diferente forma en función del género del profesorado. Estos resultados son afines a los presentados por otros autores en España como Gutiérrez y López (2011), quienes afirman que los alumnos muestran actitudes más negativas ante las tareas propuestas por las profesoras; DelCastillo et al. (2014), quienes señalan una discriminación de género del profesorado en el uso del lenguaje al dirigirse a las alumnas y, por tanto, se implican menos, o Gil-Madrona et al. (2014), al señalar una menor participación de las alumnas en las sesiones prácticas de EF. Fuera de España, en el contexto europeo, Hopf y Hatzichristou (1999), en Francia, y Trouilloud et al. (2002), en Grecia, sugieren que existe menor interacciónde las profesoras con el alumnado. Esta última idea es compartida por autores como Basow(2000) o Bellamy (1993) en los Estados Unidos.

\section{IMPLICACIÓN DEL ALUMNADO EN LA TAREA}

Los resultados obtenidos indican que los alumnos se implican más que las alumnas en la realización de Habilidades deportivas propuestas por un profesor que utiliza el Mando directo para impartir un contenido técnico en primer curso (Hipótesis=1).

En apoyo de esta hipótesis, los estudios de Bennett (2000), Carlson (1995), Griffin et al. (2001) y Pascual (2011) señalan que los contenidos de la sesión, generalmente, están dirigidos hacia el rendimiento (Habilidades deportivas con carácter físico) o la competición, 
lo cual provoca una discriminación respecto a las alumnas, al implicarse ellas menos en este tipo de tareas. Así mismo, se está de acuerdo con autores como DelCastillo-Andrés et al. (2015), cuando señalan en su estudio una mayor inclusión en las programaciones didácticas de estilos de enseñanza directivos, como el Mando directo, lo cual provoca nuevamente una menor implicación de las alumnas, como se ha constatado en los datos de esta investigación. En coherencia con los resultados aquí obtenidos, se está de acuerdo con los planteamientos de Abós et al. (2015), Cañabate et al. (2014), Cuevas, García-Calvo y Contreras (2013), Granero-Gallegos y Baena-Extremera (2014) y DelCastillo-Andrés, Campos y Ries (2013), quienes señalan la necesidad de reorientar el diseño de las sesiones prácticas de EF hacia modelos más inclusivos para los dos géneros (LOMCE, 2013). A tenor de los datos de este estudio se piensa que, rediseñando la estructura y el contenido de las sesiones prácticas de EF, como plantean autores como Granero-Gallegos y Baena-Extremera (2014) y Martos et al. (2014), se puede conseguir una mayor implicación de las alumnas en dichas sesiones. Así pues, podemos señalar que los resultados de nuestro estudio en relación a la implicación del alumnado suponen un avance, y una mayor concreción, respecto a las investigaciones realizadas por los anteriores autores quienes centran su objetivo en determinar los tipos de participación o implicación del alumnado, y no tanto en el establecimiento de los factores que afectan a la misma.

En cuanto a las dos dimensiones siguientes, Solicitación de información adicional y Aceptación de tareas, se señala previamente que debido a las características concretas de dichas dimensiones, al haber sido diseñadas para esta investigación y al no contar con estudios que permitan la discusión de los datos obtenidos por estas, se discuten únicamente las especificidades encontradas a través del registro realizado.

\section{SOLICITACIÓN DE INFORMACIÓN ADICIONAL}

En función de los datos obtenidos tras el análisis e interpretación de los resultados se puede rechazar que los alumnos solicitan más información adicional que las alumnas a los profesores cuando trabajan Habilidades deportivas de contenido técnico a través de Mando directo en primer curso (Hipótesis $\neq 2$ ).

Estos datos se muestran contrarios a los presentados por Hopf y Hatzichristou (1999), Lentillon, Cogérino y Kaestner (2006) y Trouilloud et al. (2002) en el contexto europeo de Francia y Grecia, Davis (2003) y Leaper y Brown (2008) en los Estados Unidos y Duffy, Warren y Walsh (2001) en Canadá, en los que se plantea, de forma general, una mayor participación de los alumnos cuando la clase es impartida por un profesor. En este sentido, en el contexto de esta investigación, se observa un avance en materia de equidad de género en cuanto a la participación de las alumnas en las clases de EF, con lo cual cabe la posibilidad de plantear que la línea de trabajo proyectada en el I Plan Estratégico para la Igualdad de Mujeres y Hombres en Andalucía (2010-2013) es la adecuada para eliminar, o disminuir, las desigualdades de género dentro del aula de EF. Este planteamiento, está en consonancia con las conclusiones de los estudios de Cañabate et al. (2014), DelCastilloandrés et al. (2014), Valdivia-Moral et al. (2012) y Gil-Madrona et al. (2014), al plantear una reorientación del actual diseño de las sesiones prácticas de EF a través de un modelo inclusivo para las alumnas. 


\section{ACEPTACIÓN DE TAREAS}

Los resultados vinculados a esta dimensión permiten rechazar la hipótesis que plantea que las alumnas rechazan las tareas propuestas por los profesores cuando el contenido a desarrollar en la sesión práctica de EF es Habilidad deportiva de contenido físico impartida a través de Mando directo en primer curso (Hipótesis $\neq 3$ ).

De nuevo, estos datos apuntan a la necesidad no tanto del cambio de contenidos en las sesiones prácticas de EF, como a un cambio en la estructura metodológica de las mismas para la inclusión de las alumnas, idea que es apoyada por autores como Albert López et al. (2015), Delcastillo-Andrés, Campos-Mesa y Castañeda (2015) y Brown y Chu (2012).

Una limitación significativa de este estudio fue el número de centros participantes. Sin embargo, en el diseño de la investigación, y en la planificación de objetivos, se intentó detectar la realidad de las sesiones prácticas de estos centros de Sevilla, en el sur España, para poder observar correctamente los posibles comportamientos sexistas que se transmiten en la materia de EF. Si bien esta muestra nos impide generalizar los resultados, el instrumento de observación recoge información más amplia que los estudios anteriores sobre los comportamientos sexistas que se pueden dar en el aula. La utilización de esta escala de observación ayudará al profesorado a evaluar la forma en que se transmite el sexismo dentro de sus clases prácticas. Esta observación puede llevar al profesorado a que autoevalúen su docencia, planteen una reflexión crítica de sus comportamientos y reorienten su actividad docente, si fuese necesario, para reducir la discriminación que las alumnas pueden sufrir en las clases de EF.

\section{CONCLUSIONES}

Según las afirmaciones mostradas hasta ahora, se puede concluir que se establecen conductas sexistas entre el alumnado de Educación Secundaria en Sevilla (España), dentro de la sesión práctica de EF a través de la implicación del alumnado en la tarea. En este sentido, para integrar los dos géneros, se deben plantear contenidos didácticos diferentes a los tradicionales (Habilidades deportivas y Expresión corporal), ya que estos fomentan la discriminación de género. La intervención del profesorado debe fomentar la planificación de las sesiones prácticas de EF apoyándose en la Asignación de tareas y la Diversificación de niveles al favorecer, estos, la implicación de las alumnas.

En resumen, para eliminar la barrera sexista establecida a través de las conductas del alumnado, se defiende actuar sobre la implicación del alumnado en la tarea, tanto cuantitativa como cualitativamente, sobre la base de un clima motivacional integrador y significativo para las alumnas y que elimine la diferente participación en las clases de EF por género. Por su parte, la solicitación de información adicional y la aceptación de tareas no deben suponer un sesgo en la presencia y visualización de las alumnas en las clases. Actuando de esta manera se garantiza a ambos géneros la igualdad de acceso a los recursos necesarios para llevar a cabo las tareas asignadas por el profesorado en clases de EF, garantizando las mismas posibilidades de aprendizaje para los alumnos y las alumnas. 


\section{REFERENCIAS}

ABOS, Ángel et al. Motivación en educación física y su relación con la intención de práctica de actividad física. En: LARA, A; ESPEJO, N; OCAÑA, M.; CACHÓN, M. La Formación Integral a través del Deporte. Jaén, 2015. p. 313-323.

ACKER, Ragnar Van et al. Sex equity and physical activity levels in coeducational physical education: exploring the potential of modified game forms. Physical Education and Sport Pedagogy,

Chichester, v. 15, p. 159-173, 2010.

ALBERT LÓPEZ, Nadal; CAMERINO FOGUET, Oleguer; CASTAÑER BALCELLS, Marta. Evaluar la motivación en la educación física, una aplicación con AMPET. Tándem: Didáctica de la Educacion Física, Barcelona, v. 47, p. 55-64, 2015.

BASOW, Susan A. Best and worst professors: gender patterns in students' choices. Sex Roles, Connecticut, v. 43, n. 5-6, p. 407-417, 2000.

BELLAMY, Nedaro. Bias in the classroom: are we guilty? Science Scope, Massachusetts, v. 17, n. 6, p. 60-63, 30 nov. 1993.

BENNETT, Gregg. Students' participation styles in two university weight training classes. Journal of Teaching in Physical Education, Champaign, v. 19, p. 182-205, 2000.

BRONFENBRENNER, Urie. Environments in developmental perspectrive: theoretical and operational models. In: Measuring environment across the life span: Emerging methods and concepts. [s.l: s.n.], 1999.

BROWN, Christia Spears; CHU, Hui. Discrimination, ethnic identity, and academic outcomes of Mexican immigrant children: the importance of school context. Child development, Chichester, v. 83, n. 5, p. 1477-1485, jan. 2012.

CAÑABATE ORTÍZ, Dolors et al. Perfiles motivacionales en las sesiones de educación física (Motivational profiles in physical education sessions). RETOS: Nuevas Tendencias en Educación Física, Deporte y Recreación, Murcia, v. 26, p. 34-39, 2014.

CARLSON, Teresa. "Now, I think I can." The reaction of eight low-skilled students to sport education. ACHPER Healthy Lifestyles Journal, Hindmarsh, v. 42, p. 6-8, 1995.

CASTELLANO PAULIS, Julen; HERNÁNDEZ MENDO, Antonio. La observación aplicada en diferentes ámbitos de la actividad física y el deporte: los fundamentos de base. Revista Española de Educación Física Deportes, Madrid, v. 409, p. 15-19, 2015.

CHALABAEV, Aina et al. The influence of sex stereotypes and gender roles on participation and performance in sport and exercise: Review and future directions. Psychology of Sport and Exercise, Amsterdam, v. 14, p. 136-144, 2013.

CUEVAS-CAMPOS, Ricardo; GARCÍA-CALVO, Tomás; CONTRERAS, Onofre. Perfiles motivacionales en Educación Física: una aproximación desde la teoría de las Metas de Logro 2x2. Anales de Psicología, Murcia, v. 29, n. 3, p. 685-692, 1 out. 2013.

DAVIS, Kathryn. Teaching for gender equity in physical education: a review of the literature. Women in Sport \& Physical Activity Journal, Stanningley, v. 12, p. 55-81, 2003.

DE LA CRUZ, E. et al. Consumo de alcohol durante fines de semana en universitarios almerienses activos en función del carácter de la modalidad deportiva practicada. Cultura, Ciencia y Deporte, Murcia, v. 3, p. 100, 2008. 
DELCASTILLO-ANDRÉS, Óscar et al. Gender equity in physical education: The use of language. Motriz: Revista de Educación Física, Río Claro, v. 20, n. 3, p. 239-248, set. 2014.

DELCASTILLO-ANDRES, Óscar; CORRAL PERNÍA, Juan-Antonio. El profesorado frente a la discriminación de género: uso de la retroalimentación. Cultura y Educación: Culture and Education, Barcelona, v. 23:4, n. 1578-4118, p. 487-498, 23 jan. 2011.

DELCASTILLO-ANDRÉS, Óscar; ROMERO GRANADOS, Santiago. Gender equity in Physical Education: the use of information. Sex roles, Connecticut, v. 67, p. 108-121, 2012.

DELCASTILLO-ANDRÉS, Óscar. Evaluación de los factores psicosociales y didácticos relacionados con la equidad de género en Educación Física. Sevilla: Universidad de Sevilla, 31 dez. 2009.

DELCASTILLO-ANDRÉS, Óscar; CAMPOS MESA, María del Carmen; CASTAÑEDA, Carolina. La formación del profesorado en Educación Física. En: LARA, A; ESPEJO, N; OCAÑA, M.; CACHÓN, M. La Formación Integral a través del Deporte. Jaén, 2015. p. 103-138.

DELCASTILLO-ANDRÉS, Óscar; CAMPOS MESA, María del Carmen; FRANCIS, Ries. Gender equality in Physical Education from the perspective of Achievement Goal Theory. Journal of Sport and Health Research, Jaén, v. 5, n. 1, p. 57-70, 2013.

DUFFY, Jim; WARREN, Kelly; WALSH, Margaret. Classroom interactions: Gender of teacher, gender of student, and classroom subject. Sex roles, Connecticut, v. 45, p. 579-593, 2001.

DYSON, Ben P.; RHODES LINEHAN, Nicole; HASTIE, Peter A. The ecology of cooperative learning in elementary physical education classes. Journal of Teaching in Physical Education, Champaign, v. 29, p. 113-130, 2010.

GIL-MADRONA, Pedro et al. Las niñas también quieren jugar: la participación conjunta de niños y niñas en actividades físicas no organizadas en el contexto escolar. Movimento, Porto Alegre, v. 20, p. 103-124, 2014.

GRANERO GALLEGOS, Antonio; BAENA EXTREMERA, Antonio. Predicción de la motivación autodeterminada según las orientaciones de meta y el clima motivacional en Educación Física. Retos: nuevas tendencias en nuevas tendencias en educación física, deporte y recreación, Murcia, v. 25, p. 23-27, 2014.

GRIFFIN, L. L. et al. Chapter 4. Middle school students' conceptions of soccer: their solutions to tactical problems. Journal of Teaching in Physical Education, Champaign, v. 20, n. 4, p. 324-340, 2001.

GRIFFIN, Patricia S. Girls' participation patterns in a middle school team sports unit. Journal of Teaching in Physical Education, Champaign, v. 4, p. 30-38, 1984.

GRIFFIN, Patricia S. Boys' participation styles in a middle school physical education team sports unit. Journal of Teaching in Physical Education, Champaign, v. 4, p. 100-110, 1985.

GUTIÉRREZ SANMARTÍN, Melchor; LÓPEZ, Esther. Percepción de las estrategias que emplean los profesores para mantener la disciplina, razones de los alumnos para ser disciplinados y comportamiento en Educacion Física. RICYDE, Revista Internacional de Ciencias del Deporte, Murcia, v. 22, p. 24-38, 2011.

HOPF, Diether; HATZICHRISTOU, Chryse. Teacher gender-related influences in Greek schools. British Journal of Educational Psychology, Chichester, v. 69, n. 1, p. 1-18, 16 mar. 1999.

INSTITUTO ANDALUZ DE LA MUJER. I Plan estratégico para la igualdad de mujeres y hombres en Andalucía: 2010-2013. Sevilla: Consejería para la igualdad y el bienestar social, 2010. 
LEAPER, Campbell; BROWN, Christia Spears. Perceived experiences with sexism among adolescent girls. Child development, Chichester, v. 79, n. 3, p. 685-704, jan. 2008.

LENTILLON, Vanessa; COGÉRINO, Geneviève; KAESTNER, Mattias. Injustice in physical education: gender and the perception of deprivation in grades and teacher support. Social Psychology of Education, Cham, v. 9, n. 3, p. 321-339, ago. 2006.

LOMCE, De. Ley Orgánica 8/2013, de 9 de diciembre, para la mejora de la calidad educativaBoletín Oficial del Estado. 2013. Disponível em: <http://www.stecyl.es/LOMCE/LOELOMCE.pdf>. Acesso em: 4 maio. 2015

MARTOS GARCIA, Daniel et al. El desarrollo de la autonomía y la responsabilidad en educación física: Un estudio de caso colaborativo en secundaria. Retos: nuevas tendencias en nuevas tendencias en educación física, deporte y recreación, Murcia, v. 26, p. 3-8, 2014.

MORA, Ángel; CRUZ, Jaume; SOUSA, Catarina. Cómo mejorar el clima motivacional y los estilos de comunicación en el ámbito de la Educación Física y el deporte. Infancia y Aprendizaje, Barcelona, v. 36, p. 91-103, 2013.

SALKIND, Neil J. Métodos de investigación. México: Pearson Educación, 1999.

PASCUAL, Isabel. Manual del tecnico deportivo. Zaragoza: Mira, 2011.

PREWITT-FREILINO, Jennifer; CASWELL, T. Andrew; LAAKSO, Emmi K. The gendering of language: A comparison of gender equality in countries with gendered, natural gender, and genderless languages. Sex roles, Connecticut, v. 66, p. 268-281, 2012.

PRIES DEVIDE, Fabiano et al. Estudos de gênero na educação física brasileira. Motriz, Rio Claro, v. 17, p. 93-103, 2011.

SANTOS, Vilma Canazart dos. Indícios de sentidos e significados de feminilidade e masculinidade em aulas de educação física. Motriz, Rio Claro, v. 16, p. 841-852, 2010.

TROUILLOUD, David. et al. The influence of teacher expectations on student achievement in physical education classes: Pygmalion revisited. European Journal of Social Psychology, Chichester, v. 32, n. 5, p. 591-607, 2002.

VALDIVIA-MORAL, Pedro A. et al. Concept of coeducation in Physical Education teachers and the methodology followed in its practice. Movimento, Porto Alegre, v. 18, n. 4, p. 197-217, 2012. 


\section{APÉNDICE}

Categorías de codificación

\begin{tabular}{|c|c|c|c|}
\hline & Implicación en la tarea & $\begin{array}{c}\text { Solicitación de } \\
\text { información adicional }\end{array}$ & Aceptación de tareas \\
\hline $\begin{array}{l}\text { Núcleo } \\
\text { categorial }\end{array}$ & $\begin{array}{l}\text { Se refiere a la actitud del alumnado } \\
\text { hacia la actividad que va a realizar en } \\
\text { cuanto al grado de cooperación en la } \\
\text { tarea propuesta (coopera con los dos } \\
\text { géneros, no coopera con los dos gé- } \\
\text { neros e invisible). Entenderemos que } \\
\text { una actitud es cooperativa con los dos } \\
\text { géneros cuando colabora en la organi- } \\
\text { zación de las actividades y realiza la } \\
\text { tarea con compañeros de ambos gé- } \\
\text { neros. Por el contrario, la actitud será } \\
\text { no cooperativa si observamos que el } \\
\text { alumnado se niega a cooperar con el } \\
\text { otro género, perturbando el desarro- } \\
\text { llo normal de la clase. Se constatará } \\
\text { la conducta del alumnado como invi- } \\
\text { sible cuando, aparentemente, esté } \\
\text { participando en la tarea. Asimismo, se } \\
\text { relaciona la implicación con el tipo de } \\
\text { ropa que utiliza (deportiva o no) y su } \\
\text { aportación de ideas en la tarea. }\end{array}$ & $\begin{array}{l}\text { Se entenderá que el } \\
\text { alumnado solicita este } \\
\text { tipo de información } \\
\text { cuando haga pregun- } \\
\text { tas al profesorado o de- } \\
\text { mande una ampliación } \\
\text { de la explicación dada } \\
\text { por este en cualquier } \\
\text { momento de la clase. } \\
\text { La información solicita- } \\
\text { da debe tener relación } \\
\text { con la tarea que se } \\
\text { está realizando. }\end{array}$ & $\begin{array}{l}\text { Entenderemos la aceptación } \\
\text { de tareas como la aproba- } \\
\text { ción por parte del alumnado } \\
\text { hacia la tarea propuesta he- } \\
\text { cha por el profesorado. Esto } \\
\text { implica que, posteriormente } \\
\text { a la información impartida, } \\
\text { no existe ninguna discusión } \\
\text { evasiva sobre la tarea que } \\
\text { tiene que realizar. }\end{array}$ \\
\hline Propósito & $\begin{array}{l}\text { Comprobar la disposición del alumna- } \\
\text { do hacia una tarea concreta y ver la } \\
\text { relación entre la implicación del alum- } \\
\text { nado (cooperación, ropa y aportación } \\
\text { de ideas), el tipo de tareas (técnicas } \\
\text { o físicas) y el género. Nos interesa } \\
\text { conocer los niveles de implicación del } \\
\text { alumnado ante diferentes bloques de } \\
\text { contenidos. }\end{array}$ & $\begin{array}{l}\text { Pretendemos deter- } \\
\text { minar si existe mayor } \\
\text { interés por una amplia- } \\
\text { ción de la información } \\
\text { de forma diferenciada } \\
\text { según género. }\end{array}$ & $\begin{array}{l}\text { Se pretende comprobar la } \\
\text { respuesta de agrado o des- } \\
\text { agrado, aceptación o recha- } \\
\text { zo que manifiesta uno y otro } \\
\text { sexo ante las diferentes ta- } \\
\text { reas que son propuestas en } \\
\text { una sesión y el tipo de tarea, } \\
\text { física o técnica, al que hacen } \\
\text { referencia. }\end{array}$ \\
\hline Registro & $\begin{array}{l}\text { Se registrará el tipo de cooperación } \\
\text { del alumnado, cuántos de ellos/as no } \\
\text { llevan ropa deportiva, el número de } \\
\text { ideas de apoyo hacia actitudes equi- } \\
\text { tativas con los compañeros aportadas } \\
\text { por ellos/as, el contenido de la tarea } \\
\text { que vamos a realizar y el género de } \\
\text { los sujetos observados en estas con- } \\
\text { ductas. }\end{array}$ & $\begin{array}{l}\text { Se contabilizará el nú- } \\
\text { mero de veces que chi- } \\
\text { cos y/o chicas solicitan } \\
\text { este tipo de informa- } \\
\text { ción al profesorado a lo } \\
\text { largo de la clase. }\end{array}$ & $\begin{array}{l}\text { Se registrará el número de } \\
\text { comportamientos verbales } \\
\text { (expresiones individuales, co- } \\
\text { mentarios a compañeros etc.) } \\
\text { o gestuales, apreciable en la } \\
\text { observación, procedente de } \\
\text { cualquier alumno o alumna } \\
\text { que manifieste una acepta- } \\
\text { ción o rechazo ante las tareas } \\
\text { propuestas. Indicaremos, a su } \\
\text { vez, si la tarea es de tipo físico } \\
\text { o técnico. }\end{array}$ \\
\hline Procedimiento & $\begin{array}{l}\text { Marcaremos en la escala de observa- } \\
\text { ción, el tipo de tarea mediante una } F \\
\text { (físico) o T (técnico) dentro de la casi- } \\
\text { lla correspondiente a cooperación, no } \\
\text { cooperación o invisible cada vez que } \\
\text { un alumno o una alumna desarrolle } \\
\text { una conducta de las señaladas. Así } \\
\text { mismo, señalaremos con una X cada } \\
\text { vez que un alumno o una alumna del } \\
\text { grupo aporten alguna idea de carác- } \\
\text { ter actitudinal. En el caso de la ropa } \\
\text { deportiva contabilizaremos el número } \\
\text { de alumnos y de alumnas que no cum- } \\
\text { plen este parámetro. }\end{array}$ & $\begin{array}{l}\text { Una vez finalizada la } \\
\text { información inicial apor- } \\
\text { tada por el profesorado } \\
\text { ante cada tarea, se } \\
\text { contabilizarán, mediante } \\
\text { una X en la casilla co- } \\
\text { rrespondiente, cada vez } \\
\text { que se solicita informa- } \\
\text { ción adicional por parte } \\
\text { de los alumnos o las } \\
\text { alumnas hasta la finali- } \\
\text { zación de la sesión. }\end{array}$ & $\begin{array}{l}\text { Marcaremos con una } X \text { en } \\
\text { las casillas correspondientes } \\
\text { a alumno o alumna, cada vez } \\
\text { que se produzca la acepta- } \\
\text { ción o rechazo a las tareas } \\
\text { propuestas por el profesora- } \\
\text { do, indicando el tipo de tarea } \\
\text { que tiene que desarrollar } \\
\text { según su contenido físico o } \\
\text { técnico. }\end{array}$ \\
\hline
\end{tabular}

